\title{
On the bounds for the spectral norms of geometric circulant matrices
}

\section{Can Kızılateş ${ }^{1 *}$ and Naim Tuglu²}

${ }^{\text {*Correspondence: }}$ cankizilates@gmail.com

${ }^{1}$ Department of Mathematics, Bülent Ecevit University, Zonguldak, Turkey

Full list of author information is available at the end of the article

\section{Abstract}

In this paper, we define a geometric circulant matrix whose entries are the generalized Fibonacci numbers and hyperharmonic Fibonacci numbers. Then we give upper and lower bounds for the spectral norms of these matrices.

MSC: Primary 15A60; 11B39; secondary 15B05

Keywords: generalized Fibonacci number; hyperharmonic Fibonacci number; geometric circulant matrix; spectral norms

\section{Introduction}

The circulant and $r$-circulant matrices have important applications in numerical analysis, probability, coding theory, and so on. An $n \times n$ matrix $C_{r}$ is called an $r$-circulant matrix if it is defined as follows:

$$
C_{r}=\left(\begin{array}{cccccc}
c_{0} & c_{1} & c_{2} & \ldots & c_{n-2} & c_{n-1} \\
r c_{n-1} & c_{0} & c_{1} & \ldots & c_{n-3} & c_{n-2} \\
r c_{n-2} & r c_{n-1} & c_{0} & \ldots & c_{n-4} & c_{n-3} \\
\vdots & \vdots & \vdots & & \vdots & \vdots \\
r c_{1} & r c_{2} & r c_{3} & \ldots & r c_{n-1} & c_{0}
\end{array}\right) .
$$

The matrix $C_{r}$ is determined by its first row elements and $r$, we denote $C_{r}=\operatorname{Circ}_{r}\left(c_{0}, c_{1}, c_{2}\right.$, $\left.\ldots, c_{n-1}\right)$. In particular for $r=1$

$$
C=\left(\begin{array}{cccccc}
c_{0} & c_{1} & c_{2} & \ldots & c_{n-2} & c_{n-1} \\
c_{n-1} & c_{0} & c_{1} & \ldots & c_{n-3} & c_{n-2} \\
c_{n-2} & c_{n-1} & c_{0} & \ldots & c_{n-4} & c_{n-3} \\
\vdots & \vdots & \vdots & & \vdots & \vdots \\
c_{1} & c_{2} & c_{3} & \ldots & c_{n-1} & c_{0}
\end{array}\right)
$$

is called a circulant matrix.

Circulant and $r$-circulant matrices with the special numbers have been studied by many researchers in last decade. For example, in [1], Solak has studied the spectral norms of circulant matrices with the Fibonacci and Lucas numbers. In [2], Kocer et al. obtained norms of circulant and semicirculant matrices with Horadam numbers. In [3], Shen and

(c) The Author(s) 2016. This article is distributed under the terms of the Creative Commons Attribution 4.0 International License (http://creativecommons.org/licenses/by/4.0/), which permits unrestricted use, distribution, and reproduction in any medium, provided you give appropriate credit to the original author(s) and the source, provide a link to the Creative Commons license, and indicate if changes were made. 
Cen have given upper and lower bounds for the spectral norms of $r$-circulant matrices with the Fibonacci and Lucas numbers. In [4], Bahsi computed the spectral norms of circulant and $r$-circulant matrices with the hyperharmonic numbers. Moreover, in [5], Bahsi and Solak studied norms of circulant and $r$-circulant matrices with the hyper-Fibonacci and hyper-Lucas numbers. In [6], Jiang and Zhou studied spectral norms of even order $r$ circulant matrices. In [7, 8], Tuglu and Kızılateş have calculated Euclidean norm by using the finite difference operator and given spectral norms of circulant, $r$-circulant and some special matrices with the harmonic Fibonacci and hyperharmonic Fibonacci numbers. In [9], Yazlık and Taskara have presented new upper and lower bounds for the spectral norms of an $r$-circulant matrix with the generalized $k$-Horadam numbers. In [10], He et al. gave the upper bound estimation of the spectral norm for $r$-circulant matrices with Fibonacci and Lucas numbers.

In view of the above papers, we define a new circulant matrix which is called geometric circulant matrix and give upper and lower bounds for the spectral norms of this matrix with the generalized Fibonacci and hyperharmonic Fibonacci numbers by using the same method given in [3].

\section{Preliminaries}

The well-known Fibonacci and Lucas sequences are defined by the following recurrence relations: for $n \geq 0$,

$$
F_{n+2}=F_{n+1}+F_{n}
$$

and

$$
L_{n+2}=L_{n+1}+L_{n}
$$

where $F_{0}=0, F_{1}=1, L_{0}=2$ and $L_{1}=1$, respectively. The generalized Fibonacci and Lucas sequences, $\left\{U_{n}\right\}$ and $\left\{V_{n}\right\}$, are defined by the following recurrence relations: for $n \geq 0$, and any non-zero integer $p$,

$$
U_{n+2}=p U_{n+1}+U_{n}
$$

and

$$
V_{n+2}=p V_{n+1}+V_{n},
$$

where $U_{0}=0, U_{1}=1, V_{0}=2$ and $V_{1}=p$. If we take $p=1$, then $U_{n}=F_{n}$ and $V_{n}=L_{n}$. Let $\alpha$ and $\beta$ be the roots of the characteristic equation $x^{2}-p x-1=0$. Then the Binet formulas for the sequences $\left\{U_{n}\right\}$ and $\left\{V_{n}\right\}$ are given by

$$
U_{n}=\frac{\alpha^{n}-\beta^{n}}{\alpha-\beta}
$$

and

$$
V_{n}=\alpha^{n}+\beta^{n},
$$

where $\alpha=\frac{p+\sqrt{p^{2}+4}}{2}$ and $\beta=\frac{p-\sqrt{p^{2}+4}}{2}$. 
On the other hand, Yazlık and Taskara examined the generalized $k$-Horadam numbers via the following recurrence relations:

$$
H_{k, n+2}=f(k) H_{k, n+1}+g(k) H_{k, n}
$$

with the initial values $H_{k, 0}=a, H_{k, 1}=b$. Moreover they calculated sum of squares of $k$ Horadam numbers (see [9]). If we take $f(k)=p, g(k)=1, a=0$ and $b=1$ in (1), we get

$$
\sum_{i=0}^{n-1} U_{i}^{2}=\frac{U_{n}^{2}-U_{n-1}^{2}+(-1)^{n}}{p^{2}}
$$

and if we take $f(k)=p, g(k)=1, a=2$ and $b=p$ in (1), we have

$$
\sum_{i=0}^{n-1} V_{i}^{2}=\frac{V_{n}^{2}-V_{n-1}^{2}+p^{2}-4+\left(1-(-1)^{n}\right)\left(p^{2}+4\right)}{p^{2}} .
$$

In [11], Tuglu et al. defined hyperharmonic Fibonacci numbers for $n, r \geq 1$,

$$
\mathbb{F}_{n}^{(r)}=\sum_{k=1}^{n} \mathbb{F}_{k}^{(r-1)}
$$

where $\mathbb{F}_{n}^{(0)}=\frac{1}{F_{n}}$ and $\mathbb{F}_{0}=0$. Then they gave for the sum of the squares of hyperharmonic Fibonacci numbers as follows:

$$
\frac{1}{\sqrt{n}} \mathbb{F}_{n-1}^{(r+1)} \leq \sqrt{\sum_{k=0}^{n-1}\left(\mathbb{F}_{k}^{(r)}\right)^{2}} \leq \mathbb{F}_{n-1}^{(r+1)}
$$

Now we give some definitions and Lemmas related to our study.

Definition 1 An $n \times n$ matrix $C_{r^{*}}$ is called a geometric circulant matrix if it is of the form

$$
C_{r^{*}}=\left(\begin{array}{cccccc}
c_{0} & c_{1} & c_{2} & \ldots & c_{n-2} & c_{n-1} \\
r c_{n-1} & c_{0} & c_{1} & \ldots & c_{n-3} & c_{n-2} \\
r^{2} c_{n-2} & r c_{n-1} & c_{0} & \ldots & c_{n-4} & c_{n-3} \\
\vdots & \vdots & \vdots & & \vdots & \vdots \\
r^{n-1} c_{1} & r^{n-2} c_{2} & r^{n-3} c_{3} & \ldots & r c_{n-1} & c_{0}
\end{array}\right) .
$$

We denote it for brevity by $C_{r^{*}}=\operatorname{Circ}_{r^{*}}\left(c_{0}, c_{1}, c_{2}, \ldots, c_{n-1}\right)$. Note that, for $r=1$, geometric circulant matrix turns into circulant matrix given in $[11,12]$. In fact, in $[11,12]$, the authors calculated the spectral norms of the circulant matrices with the generalized Fibonacci and hyperharmonic Fibonacci numbers.

Definition 2 Let $A=\left(a_{i j}\right)$ be any $m \times n$ matrix. The Euclidean norm of matrix $A$ is

$$
\|A\|_{E}=\sqrt{\left(\sum_{i=1}^{m} \sum_{j=1}^{n}\left|a_{i j}\right|^{2}\right)} .
$$


Definition 3 Let $A=\left(a_{i j}\right)$ be any $m \times n$ matrix. The spectral norm of matrix $A$ is

$$
\|A\|_{2}=\sqrt{\max _{1 \leq i \leq n} \lambda_{i}\left(A^{H} A\right)},
$$

where $\lambda_{i}\left(A^{H} A\right)$ is eigenvalue of $A^{H} A$ and $A^{H}$ is conjugate transpose of matrix $A$.

Then the following inequalities hold between the Euclidean norm and the spectral norm:

$$
\begin{aligned}
& \frac{1}{\sqrt{n}}\|A\|_{E} \leq\|A\|_{2} \leq\|A\|_{E}, \\
& \|A\|_{2} \leq\|A\|_{E} \leq \sqrt{n}\|A\|_{2} .
\end{aligned}
$$

Definition 4 Let $A=\left(a_{i j}\right)$ and $B=\left(b_{i j}\right)$ are each $m \times n$ matrices, then their Hadamard product is the $m \times n$ matrix of elementwise products

$$
A \circ B=\left(a_{i j} b_{i j}\right)
$$

Lemma 1 [13] Let $A$ and $B$ be two $m \times n$ matrices. We have

$$
\|A \circ B\|_{2} \leq r_{1}(A) c_{1}(B)
$$

where

$$
\begin{aligned}
& r_{1}(A)=\max _{1 \leq i \leq m} \sqrt{\sum_{j=1}^{n}\left|a_{i j}\right|^{2}}, \\
& c_{1}(B)=\max _{1 \leq j \leq n} \sqrt{\sum_{i=1}^{m}\left|b_{i j}\right|^{2}} .
\end{aligned}
$$

\section{Main results}

Theorem 1 Let $U_{r^{*}}=\operatorname{Circ}_{r^{*}}\left(U_{0}, U_{1}, U_{2}, \ldots, U_{n-1}\right)$ be an $n \times n$ geometric circulant matrix.

(i) If $|r|>1$, then

$$
\sqrt{\frac{U_{n}^{2}-U_{n-1}^{2}+(-1)^{n}}{p^{2}}} \leq\left\|U_{r^{*}}\right\|_{2} \leq \sqrt{\frac{\left(|r|^{2}-|r|^{2 n}\right)\left(U_{n}^{2}-U_{n-1}^{2}+(-1)^{n}\right)}{\left(1-|r|^{2}\right) p^{2}}}
$$

(ii) If $|r|<1$, then

$$
\begin{aligned}
& \frac{|r|}{\sqrt{p^{2}+4}} \sqrt{\frac{2|r|^{2 n+2}-|r|^{2 n}\left(p^{2}+2\right)-|r|^{2} V_{2 n}+V_{2 n-2}}{|r|^{4}-|r|^{2}\left(p^{2}+2\right)+1}-2 \frac{|r|^{2 n}-(-1)^{n}}{|r|^{2}+1}} \\
& \leq\left\|U_{r^{*}}\right\|_{2} \leq \sqrt{\frac{(n-1)\left(U_{n}^{2}-U_{n-1}^{2}+(-1)^{n}\right)}{p^{2}}}
\end{aligned}
$$


Proof We have the matrix

$$
U_{r^{*}}=\left(\begin{array}{cccccc}
U_{0} & U_{1} & U_{2} & \ldots & U_{n-2} & U_{n-1} \\
r U_{n-1} & U_{0} & U_{1} & \ldots & U_{n-3} & U_{n-2} \\
r^{2} U_{n-2} & r U_{n-1} & U_{0} & \ldots & U_{n-4} & U_{n-3} \\
\vdots & \vdots & \vdots & & \vdots & \vdots \\
r^{n-1} U_{1} & r^{n-2} U_{2} & r^{n-3} U_{3} & \ldots & r U_{n-1} & U_{0}
\end{array}\right)
$$

(i) From $|r|>1$ and definition of Euclidean norm, we have

$$
\begin{aligned}
\left\|U_{r^{*}}\right\|_{E}^{2} & =\sum_{k=0}^{n-1}(n-k) U_{k}^{2}+\sum_{k=1}^{n-1} k\left|r^{n-k}\right|^{2} U_{k}^{2} \\
& \geq \sum_{k=0}^{n-1}(n-k) U_{k}^{2}+\sum_{k=1}^{n-1} k U_{k}^{2} \\
& =n \sum_{k=0}^{n-1} U_{k}^{2} \\
& =n \frac{U_{n}^{2}-U_{n-1}^{2}+(-1)^{n}}{p^{2}}
\end{aligned}
$$

that is,

$$
\frac{1}{\sqrt{n}}\left\|U_{r^{*}}\right\|_{E} \geq \sqrt{\frac{U_{n}^{2}-U_{n-1}^{2}+(-1)^{n}}{p^{2}}}
$$

from (5), we have

$$
\sqrt{\frac{U_{n}^{2}-U_{n-1}^{2}+(-1)^{n}}{p^{2}}} \leq\left\|U_{r^{*}}\right\|_{2}
$$

On the other hand, let the matrices $A$ and $B$ be defined by

$$
A=\left(\begin{array}{cccccc}
U_{0} & 1 & 1 & \ldots & 1 & 1 \\
r & U_{0} & 1 & \ldots & 1 & 1 \\
r^{2} & r & U_{0} & \ldots & 1 & 1 \\
\vdots & \vdots & \vdots & & \vdots & \vdots \\
r^{n-1} & r^{n-2} & r^{n-3} & \ldots & r & U_{0}
\end{array}\right)
$$

and

$$
B=\left(\begin{array}{cccccc}
U_{0} & U_{1} & U_{2} & \ldots & U_{n-2} & U_{n-1} \\
U_{n-1} & U_{0} & U_{1} & \ldots & U_{n-3} & U_{n-2} \\
U_{n-2} & U_{n-1} & U_{0} & \ldots & U_{n-4} & U_{n-3} \\
\vdots & \vdots & \vdots & & \vdots & \vdots \\
U_{1} & U_{2} & U_{3} & \ldots & U_{n-1} & U_{0}
\end{array}\right) .
$$


That is, $U_{r^{*}}=A \circ B$. Then we obtain

$$
\begin{aligned}
r_{1}(A) & =\max _{1 \leq i \leq n} \sqrt{\sum_{j=1}^{n}\left|a_{i j}\right|^{2}} \\
& =\sqrt{\left|r^{1}\right|^{2}+\cdots+\left|r^{n-1}\right|^{2}} \\
& =\sqrt{\frac{|r|^{2}-|r|^{2 n}}{1-|r|^{2}}}
\end{aligned}
$$

and

$$
\begin{aligned}
c_{1}(B) & =\max _{1 \leq j \leq n} \sqrt{\sum_{i=1}^{n}\left|b_{i j}\right|^{2}} \\
& =\sqrt{\sum_{k=0}^{n-1} U_{k}^{2}} \\
& =\sqrt{\frac{U_{n}^{2}-U_{n-1}^{2}+(-1)^{n}}{p^{2}}} .
\end{aligned}
$$

From Lemma 1, we have

$$
\left\|U_{r^{*}}\right\|_{2} \leq \sqrt{\frac{\left(|r|^{2}-|r|^{2 n}\right)\left(U_{n}^{2}-U_{n-1}^{2}+(-1)^{n}\right)}{\left(1-|r|^{2}\right) p^{2}}}
$$

Thus, we have

$$
\sqrt{\frac{U_{n}^{2}-U_{n-1}^{2}+(-1)^{n}}{p^{2}}} \leq\left\|U_{r^{*}}\right\|_{2} \leq \sqrt{\frac{\left(|r|^{2}-|r|^{2 n}\right)\left(U_{n}^{2}-U_{n-1}^{2}+(-1)^{n}\right)}{\left(1-|r|^{2}\right) p^{2}}} .
$$

(ii) From $|r|<1$, we have

$$
\begin{aligned}
\left\|U_{r^{*}}\right\|_{E}^{2} & =\sum_{k=0}^{n-1}(n-k) U_{k}^{2}+\sum_{k=1}^{n-1} k\left|r^{n-k}\right|^{2} U_{k}^{2} \\
& \geq \sum_{k=0}^{n-1}(n-k)\left|r^{n-k}\right|^{2} U_{k}^{2}+\sum_{k=1}^{n-1} k\left|r^{n-k}\right|^{2} U_{k}^{2} \\
& =n|r|^{2 n} \sum_{k=0}^{n-1}\left(\frac{U_{k}}{|r|^{k}}\right)^{2} \\
& =\frac{n|r|^{2 n}}{p^{2}+4} \sum_{k=0}^{n-1}\left(\frac{\alpha^{k}-\beta^{k}}{|r|^{k}}\right)^{2} \\
& =\frac{n|r|^{2 n}}{p^{2}+4}\left(\frac{1-\left(\frac{\alpha^{2}}{|r|^{2}}\right)^{n}}{1-\left(\frac{\alpha^{2}}{|r|^{2}}\right)}+\frac{1-\left(\frac{\beta^{2}}{|r|^{2}}\right)^{n}}{1-\left(\frac{\beta^{2}}{|r|^{2}}\right)}-2 \frac{1-\left(\frac{-1}{|r|^{2}}\right)^{n}}{1+\frac{1}{|r|^{2}}}\right) \\
& =\frac{n|r|^{2}}{p^{2}+4} \sqrt{\frac{2|r|^{2 n+2}-|r|^{2 n}\left(p^{2}+2\right)-|r|^{2} V_{2 n}+V_{2 n-2}}{|r|^{4}-|r|^{2}\left(p^{2}+2\right)+1}-2 \frac{|r|^{2 n}-(-1)^{n}}{|r|^{2}+1}}
\end{aligned}
$$




$$
\frac{1}{\sqrt{n}}\left\|U_{r^{*}}\right\|_{E} \geq \frac{|r|}{\sqrt{p^{2}+4}} \sqrt{\frac{2|r|^{2 n+2}-|r|^{2 n}\left(p^{2}+2\right)-|r|^{2} V_{2 n}+V_{2 n-2}}{|r|^{4}-|r|^{2}\left(p^{2}+2\right)+1}-2 \frac{|r|^{2 n}-(-1)^{n}}{|r|^{2}+1}} .
$$

From (5)

$$
\frac{|r|}{\sqrt{p^{2}+4}} \sqrt{\frac{2|r|^{2 n+2}-|r|^{2 n}\left(p^{2}+2\right)-|r|^{2} V_{2 n}+V_{2 n-2}}{|r|^{4}-|r|^{2}\left(p^{2}+2\right)+1}-2 \frac{|r|^{2 n}-(-1)^{n}}{|r|^{2}+1}} \leq\left\|U_{r^{*}}\right\|_{2} .
$$

On the other hand, let the matrices $A$ and $B$ be defined by

$$
A=\left(\begin{array}{cccccc}
U_{0} & 1 & 1 & \ldots & 1 & 1 \\
r & U_{0} & 1 & \ldots & 1 & 1 \\
r^{2} & r & U_{0} & \ldots & 1 & 1 \\
\vdots & \vdots & \vdots & & \vdots & \vdots \\
r^{n-1} & r^{n-2} & r^{n-3} & \ldots & r & U_{0}
\end{array}\right)
$$

and

$$
B=\left(\begin{array}{cccccc}
U_{0} & U_{1} & U_{2} & \ldots & U_{n-2} & U_{n-1} \\
U_{n-1} & U_{0} & U_{1} & \ldots & U_{n-3} & U_{n-2} \\
U_{n-2} & U_{n-1} & U_{0} & \ldots & U_{n-4} & U_{n-3} \\
\vdots & \vdots & \vdots & & \vdots & \vdots \\
U_{1} & U_{2} & U_{3} & \ldots & U_{n-1} & U_{0}
\end{array}\right)
$$

That is, $U_{r^{*}}=A \circ B$. Then we obtain

$$
\begin{aligned}
r_{1}(A) & =\max _{1 \leq i \leq n} \sqrt{\sum_{j=1}^{n}\left|a_{i j}\right|^{2}} \\
& =\sqrt{U_{0}^{2}+n-1} \\
& =\sqrt{n-1}
\end{aligned}
$$

and

$$
\begin{aligned}
c_{1}(B) & =\max _{1 \leq j \leq n} \sqrt{\sum_{i=1}^{n}\left|b_{i j}\right|^{2}} \\
& =\sqrt{\sum_{k=0}^{n-1} U_{k}^{2}} \\
& =\sqrt{\frac{U_{n}^{2}-U_{n-1}^{2}+(-1)^{n}}{p^{2}}} .
\end{aligned}
$$

Hence, from Lemma 1, we have

$$
\left\|U_{r^{*}}\right\|_{2} \leq \sqrt{\frac{(n-1)\left(U_{n}^{2}-U_{n-1}^{2}+(-1)^{n}\right)}{p^{2}}} .
$$


Thus, we have

$$
\begin{aligned}
& \frac{|r|}{\sqrt{p^{2}+4}} \sqrt{\frac{2|r|^{2 n+2}-|r|^{2 n}\left(p^{2}+2\right)-|r|^{2} V_{2 n}+V_{2 n-2}}{|r|^{4}-|r|^{2}\left(p^{2}+2\right)+1}-2 \frac{|r|^{2 n}-(-1)^{n}}{|r|^{2}+1}} \\
& \leq\left\|U_{r^{*}}\right\|_{2} \leq \sqrt{\frac{(n-1)\left(U_{n}^{2}-U_{n-1}^{2}+(-1)^{n}\right)}{p^{2}}} .
\end{aligned}
$$

Theorem 2 Let $V_{r^{*}}=\operatorname{Circ}_{r^{*}}\left(V_{0}, V_{1}, V_{2}, \ldots, V_{n-1}\right)$ be an $n \times n$ geometric circulant matrix.

(i) If $|r|>1$, then

$$
\begin{aligned}
& \sqrt{\frac{V_{n}^{2}-V_{n-1}^{2}+p^{2}-4+\left(1-(-1)^{n}\right)\left(p^{2}+4\right)}{p^{2}}} \\
& \leq\left\|V_{r^{*}}\right\|_{2} \leq \sqrt{\frac{1-|r|^{2 n}}{1-|r|^{2}} \frac{V_{n}^{2}-V_{n-1}^{2}+p^{2}-4+\left(1-(-1)^{n}\right)\left(p^{2}+4\right)}{p^{2}}}
\end{aligned}
$$

(ii) If $|r|<1$, then

$$
\begin{aligned}
& |r| \sqrt{\frac{2|r|^{2 n+2}-|r|^{2 n}\left(p^{2}+2\right)-|r|^{2} V_{2 n}+V_{2 n-2}}{|r|^{4}-|r|^{2}\left(p^{2}+2\right)+1}+2 \frac{|r|^{2 n}-(-1)^{n}}{|r|^{2}+1}} \\
& \quad \leq\left\|V_{r^{*}}\right\|_{2} \leq \sqrt{\frac{n\left(V_{n}^{2}-V_{n-1}^{2}+p^{2}-4+\left(1-(-1)^{n}\right)\left(p^{2}+4\right)\right)}{p^{2}}} .
\end{aligned}
$$

Proof We have the matrix

$$
V_{r^{*}}=\left(\begin{array}{cccccc}
V_{0} & V_{1} & V_{2} & \ldots & V_{n-2} & V_{n-1} \\
r V_{n-1} & V_{0} & V_{1} & \ldots & V_{n-3} & V_{n-2} \\
r^{2} V_{n-2} & r V_{n-1} & V_{0} & \ldots & V_{n-4} & V_{n-3} \\
\vdots & \vdots & \vdots & & \vdots & \vdots \\
r^{n-1} V_{1} & r^{n-2} V_{2} & r^{n-3} V_{3} & \ldots & r V_{n-1} & V_{0}
\end{array}\right) .
$$

(i) From $|r|>1$, we have

$$
\begin{aligned}
\left\|V_{r^{*}}\right\|_{E}^{2} & =\sum_{k=0}^{n-1}(n-k) V_{k}^{2}+\sum_{k=1}^{n-1} k\left|r^{n-k}\right|^{2} V_{k}^{2} \\
& \geq n \sum_{k=0}^{n-1} V_{k}^{2} \\
& =\frac{n\left(V_{n}^{2}-V_{n-1}^{2}+p^{2}-4+\left(1-(-1)^{n}\right)\left(p^{2}+4\right)\right)}{p^{2}}
\end{aligned}
$$

that is,

$$
\frac{1}{\sqrt{n}}\left\|V_{r^{*}}\right\|_{E} \geq \sqrt{\frac{V_{n}^{2}-V_{n-1}^{2}+p^{2}-4+\left(1-(-1)^{n}\right)\left(p^{2}+4\right)}{p^{2}}},
$$


from (5), we have

$$
\sqrt{\frac{V_{n}^{2}-V_{n-1}^{2}+p^{2}-4+\left(1-(-1)^{n}\right)\left(p^{2}+4\right)}{p^{2}}} \leq\left\|V_{r^{*}}\right\|_{2} .
$$

On the other hand, let the matrices $A$ and $B$ be defined by

$$
A=\left(\begin{array}{cccccc}
1 & 1 & 1 & \ldots & 1 & 1 \\
r & 1 & 1 & \ldots & 1 & 1 \\
r^{2} & r & 1 & \ldots & 1 & 1 \\
\vdots & \vdots & \vdots & & \vdots & \vdots \\
r^{n-1} & r^{n-2} & r^{n-3} & \ldots & r & 1
\end{array}\right)
$$

and

$$
B=\left(\begin{array}{cccccc}
V_{0} & V_{1} & V_{2} & \ldots & V_{n-2} & V_{n-1} \\
V_{n-1} & V_{0} & V_{1} & \ldots & V_{n-3} & V_{n-2} \\
V_{n-2} & V_{n-1} & V_{0} & \ldots & V_{n-4} & V_{n-3} \\
\vdots & \vdots & \vdots & & \vdots & \vdots \\
V_{1} & V_{2} & V_{3} & \ldots & V_{n-1} & V_{0}
\end{array}\right) .
$$

That is, $V_{r^{*}}=A \circ B$. Then we obtain

$$
\begin{aligned}
r_{1}(A) & =\max _{1 \leq i \leq n} \sqrt{\sum_{j=1}^{n}\left|a_{i j}\right|^{2}} \\
& =\sqrt{1+\left|r^{1}\right|^{2}+\cdots+\left|r^{n-1}\right|^{2}} \\
& =\sqrt{\frac{1-|r|^{2 n}}{1-|r|^{2}}}
\end{aligned}
$$

and

$$
\begin{aligned}
c_{1}(B) & =\max _{1 \leq j \leq n} \sqrt{\sum_{i=1}^{n}\left|b_{i j}\right|^{2}} \\
& =\sqrt{\sum_{k=0}^{n-1} V_{k}^{2}} \\
& =\sqrt{\frac{V_{n}^{2}-V_{n-1}^{2}+p^{2}-4+\left(1-(-1)^{n}\right)\left(p^{2}+4\right)}{p^{2}}} .
\end{aligned}
$$

From Lemma 1, we have

$$
\left\|V_{r^{*}}\right\|_{2} \leq \sqrt{\frac{1-|r|^{2 n}}{1-|r|^{2}} \frac{V_{n}^{2}-V_{n-1}^{2}+p^{2}-4+\left(1-(-1)^{n}\right)\left(p^{2}+4\right)}{p^{2}}} .
$$


Thus, we have

$$
\begin{aligned}
& \sqrt{\frac{V_{n}^{2}-V_{n-1}^{2}+p^{2}-4+\left(1-(-1)^{n}\right)\left(p^{2}+4\right)}{p^{2}}} \\
& \leq\left\|V_{r^{*}}\right\|_{2} \leq \sqrt{\frac{1-|r|^{2 n}}{1-|r|^{2}} \frac{V_{n}^{2}-V_{n-1}^{2}+p^{2}-4+\left(1-(-1)^{n}\right)\left(p^{2}+4\right)}{p^{2}}} .
\end{aligned}
$$

(ii) From $|r|<1$, we have

$$
\begin{aligned}
&\left\|V_{r^{*}}\right\|_{E}^{2}=\sum_{k=0}^{n-1}(n-k) V_{k}^{2}+\sum_{k=1}^{n-1} k\left|r^{n-k}\right|^{2} V_{k}^{2} \\
& \geq \sum_{k=0}^{n-1}(n-k)\left|r^{n-k}\right|^{2} V_{k}^{2}+\sum_{k=1}^{n-1} k\left|r^{n-k}\right|^{2} V_{k}^{2} \\
&=n|r|^{2 n} \sum_{k=0}^{n-1}\left(\frac{V_{k}}{|r|^{k}}\right)^{2} \\
&=n|r|^{2 n}\left(\sum_{k=0}^{n-1} \frac{\alpha^{2 k}}{|r|^{2 k}}+\sum_{k=0}^{n-1} \frac{\beta^{2 k}}{|r|^{2 k}}+2 \sum_{k=0}^{n-1} \frac{(-1)^{k}}{|r|^{2 k}}\right) \\
&=n|r|^{2 n}\left(\frac{1-\left(\frac{\alpha^{2}}{|r|^{2}}\right)^{n}}{1-\left(\frac{\alpha^{2}}{|r|^{2}}\right)}+\frac{1-\left(\frac{\beta^{2}}{|r|^{2}}\right)^{n}}{1-\left(\frac{\beta^{2}}{|r|^{2}}\right)}+2 \frac{1-\left(\frac{-1}{|r|^{2}}\right)^{n}}{1+\frac{1}{|r|^{2}}}\right) \\
&=n|r|^{2}\left(\frac{2|r|^{2 n+2}-|r|^{2 n}\left(p^{2}+2\right)-|r|^{2} V_{2 n}+V_{2 n-2}}{|r|^{4}-|r|^{2}\left(p^{2}+2\right)+1}+2 \frac{|r|^{2 n}-(-1)^{n}}{|r|^{2}+1}\right) \\
& \frac{1}{\sqrt{n} \| V_{r^{*}}} \|_{E} \geq|r| \sqrt{\frac{2|r|^{2 n+2}-|r|^{2 n}\left(p^{2}+2\right)-|r|^{2} V_{2 n}+V_{2 n-2}}{|r|^{4}-|r|^{2}\left(p^{2}+2\right)+1}+2 \frac{|r|^{2 n}-(-1)^{n}}{|r|^{2}+1}} .
\end{aligned}
$$

From (5),

$$
|r| \sqrt{\frac{2|r|^{2 n+2}-|r|^{2 n}\left(p^{2}+2\right)-|r|^{2} V_{2 n}+V_{2 n-2}}{|r|^{4}-|r|^{2}\left(p^{2}+2\right)+1}+2 \frac{|r|^{2 n}-(-1)^{n}}{|r|^{2}+1}} \leq\left\|V_{r^{*}}\right\|_{2} \text {. }
$$

On the other hand, let the matrices $A$ and $B$ be defined by

$$
A=\left(\begin{array}{cccccc}
1 & 1 & 1 & \ldots & 1 & 1 \\
r & 1 & 1 & \ldots & 1 & 1 \\
r^{2} & r & 1 & \ldots & 1 & 1 \\
\vdots & \vdots & \vdots & & \vdots & \vdots \\
r^{n-1} & r^{n-2} & r^{n-3} & \ldots & r & 1
\end{array}\right)
$$


and

$$
B=\left(\begin{array}{cccccc}
V_{0} & V_{1} & V_{2} & \ldots & V_{n-2} & V_{n-1} \\
V_{n-1} & V_{0} & V_{1} & \ldots & V_{n-3} & V_{n-2} \\
V_{n-2} & V_{n-1} & V_{0} & \ldots & V_{n-4} & V_{n-3} \\
\vdots & \vdots & \vdots & & \vdots & \vdots \\
V_{1} & V_{2} & V_{3} & \ldots & V_{n-1} & V_{0}
\end{array}\right) .
$$

That is, $V_{r^{*}}=A \circ B$. Then we obtain

$$
r_{1}(A)=\max _{1 \leq i \leq n} \sqrt{\sum_{j=1}^{n}\left|a_{i j}\right|^{2}}=\sqrt{n}
$$

and

$$
\begin{aligned}
c_{1}(B) & =\max _{1 \leq j \leq n} \sqrt{\sum_{i=1}^{n}\left|b_{i j}\right|^{2}} \\
& =\sqrt{\sum_{k=0}^{n-1} V_{k}^{2}} \\
& =\sqrt{\frac{V_{n}^{2}-V_{n-1}^{2}+p^{2}-4+\left(1-(-1)^{n}\right)\left(p^{2}+4\right)}{p^{2}}} .
\end{aligned}
$$

From Lemma 1, we have

$$
\left\|V_{r^{*}}\right\|_{2} \leq \sqrt{\frac{n\left(V_{n}^{2}-V_{n-1}^{2}+p^{2}-4+\left(1-(-1)^{n}\right)\left(p^{2}+4\right)\right)}{p^{2}}} .
$$

Thus we have

$$
\begin{array}{r}
|r| \sqrt{\frac{2|r|^{2 n+2}-|r|^{2 n}\left(p^{2}+2\right)-|r|^{2} V_{2 n}+V_{2 n-2}}{|r|^{4}-|r|^{2}\left(p^{2}+2\right)+1}+2 \frac{|r|^{2 n}-(-1)^{n}}{|r|^{2}+1}} \\
\quad \leq\left\|V_{r^{*}}\right\|_{2} \leq \sqrt{\frac{n\left(V_{n}^{2}-V_{n-1}^{2}+p^{2}-4+\left(1-(-1)^{n}\right)\left(p^{2}+4\right)\right)}{p^{2}}} .
\end{array}
$$

Theorem 3 Let $\mathbb{F}_{r^{*}}^{(k)}=\operatorname{Circ}_{r^{*}}\left(\mathbb{F}_{0}^{(k)}, \mathbb{F}_{1}^{(k)}, \mathbb{F}_{2}^{(k)}, \ldots, \mathbb{F}_{n-1}^{(k)}\right)$ be an $n \times n$ geometric circulant matrix.

(i) If $|r|>1$, then

$$
\frac{1}{\sqrt{n}} \mathbb{F}_{n-1}^{(k+1)} \leq\left\|\mathbb{F}_{r^{*}}^{(k)}\right\|_{2} \leq \sqrt{\frac{|r|^{2}-|r|^{2 n}}{1-|r|^{2}}} \mathbb{F}_{n-1}^{(k+1)}
$$

(ii) If $|r|<1$, then

$$
\frac{|r|^{n}}{\sqrt{n}} \mathbb{F}_{n-1}^{(k+1)} \leq\left\|\mathbb{F}_{r^{*}}^{(k)}\right\|_{2} \leq \sqrt{n-1} \mathbb{F}_{n-1}^{(k+1)}
$$


Proof Since the $\mathbb{F}_{r^{*}}^{(k)}$ is of the form

$$
\mathbb{F}_{r^{*}}^{(k)}=\left(\begin{array}{cccccc}
\mathbb{F}_{0}^{(k)} & \mathbb{F}_{1}^{(k)} & \mathbb{F}_{2}^{(k)} & \ldots & \mathbb{F}_{n-2}^{(k)} & \mathbb{F}_{n-1}^{(k)} \\
r \mathbb{F}_{n-1}^{(k)} & \mathbb{F}_{0}^{(k)} & \mathbb{F}_{1}^{(k)} & \ldots & \mathbb{F}_{n-3}^{(k)} & \mathbb{F}_{n-2}^{(k)} \\
r^{2} \mathbb{F}_{n-2}^{(k)} & r \mathbb{F}_{n-1}^{(k)} & \mathbb{F}_{0}^{(k)} & \ldots & \mathbb{F}_{n-4}^{(k)} & \mathbb{F}_{n-3}^{(k)} \\
\vdots & \vdots & \vdots & & \vdots & \vdots \\
r^{n-1} \mathbb{F}_{1}^{(k)} & r^{n-2} \mathbb{F}_{2}^{(k)} & r^{n-3} \mathbb{F}_{3}^{(k)} & \ldots & r \mathbb{F}_{n-1}^{(k)} & \mathbb{F}_{0}^{(k)}
\end{array}\right)
$$

and from the definition of Euclidean norm, we have

$$
\left\|\mathbb{F}_{r^{*}}^{(k)}\right\|_{E}^{2}=\sum_{s=0}^{n-1}(n-s)\left(\mathbb{F}_{s}^{(k)}\right)^{2}+\sum_{s=1}^{n-1} s\left|r^{n-s}\right|^{2}\left(\mathbb{F}_{s}^{(k)}\right)^{2}
$$

(i) From $|r|>1$, we have

$$
\begin{aligned}
\left\|\mathbb{F}_{r^{*}}^{(k)}\right\|_{E}^{2} & \geq \sum_{s=0}^{n-1}(n-s)\left(\mathbb{F}_{s}^{(k)}\right)^{2}+\sum_{s=1}^{n-1} s\left(\mathbb{F}_{s}^{(k)}\right)^{2} \\
& =n \sum_{s=0}^{n-1}\left(\mathbb{F}_{s}^{(k)}\right)^{2} .
\end{aligned}
$$

Thus from (5) and (4),

$$
\frac{1}{\sqrt{n}} \mathbb{F}_{n-1}^{(k+1)} \leq\left\|\mathbb{F}_{r^{*}}^{(k)}\right\|_{2}
$$

On the other hand let the matrices $A^{(k)}$ and $B^{(k)}$ be defined by

$$
A^{(k)}=\left(\begin{array}{cccccc}
\mathbb{F}_{0}^{(k)} & 1 & 1 & \ldots & 1 & 1 \\
r & \mathbb{F}_{0}^{(k)} & 1 & \ldots & 1 & 1 \\
r^{2} & r & \mathbb{F}_{0}^{(k)} & \ldots & 1 & 1 \\
\vdots & \vdots & \vdots & & \vdots & \vdots \\
r^{n-1} & r^{n-2} & r^{n-3} & \ldots & r & \mathbb{F}_{0}^{(k)}
\end{array}\right)
$$

and

$$
B^{(k)}=\left(\begin{array}{cccccc}
\mathbb{F}_{0}^{(k)} & \mathbb{F}_{1}^{(k)} & \mathbb{F}_{2}^{(k)} & \ldots & \mathbb{F}_{n-2}^{(k)} & \mathbb{F}_{n-1}^{(k)} \\
\mathbb{F}_{n-1}^{(k)} & \mathbb{F}_{0}^{(k)} & \mathbb{F}_{1}^{(k)} & \ldots & \mathbb{F}_{n-3}^{(k)} & \mathbb{F}_{n-2}^{(k)} \\
\mathbb{F}_{n-2}^{(k)} & \mathbb{F}_{n-1}^{(k)} & \mathbb{F}_{0}^{(k)} & \ldots & \mathbb{F}_{n-4}^{(k)} & \mathbb{F}_{n-3}^{(k)} \\
\vdots & \vdots & \vdots & & \vdots & \vdots \\
\mathbb{F}_{1}^{(k)} & \mathbb{F}_{2}^{(k)} & \mathbb{F}_{3}^{(k)} & \ldots & \mathbb{F}_{n-1}^{(k)} & \mathbb{F}_{0}^{(k)}
\end{array}\right)
$$

That is, $\mathbb{F}_{r^{*}}^{(k)}=A^{(k)} \circ B^{(k)}$. Then we obtain

$$
r_{1}\left(A^{(k)}\right)=\max _{1 \leq i \leq n} \sqrt{\sum_{j=1}^{n}\left|a_{i j}^{(k)}\right|^{2}}=\sqrt{\frac{|r|^{2}-|r|^{2 n}}{1-|r|^{2}}}
$$


and

$$
c_{1}\left(B^{(k)}\right)=\max _{1 \leq j \leq n} \sqrt{\sum_{i=1}^{n}\left|b_{i j}^{(k)}\right|^{2}}=\sqrt{\sum_{s=0}^{n-1}\left(\mathbb{F}_{s}^{(k)}\right)^{2}} .
$$

From Lemma 1 and (4), we have

$$
\left\|\mathbb{F}_{r^{*}}^{(k)}\right\|_{2} \leq \sqrt{\frac{|r|^{2}-|r|^{2 n}}{1-|r|^{2}}} \mathbb{F}_{n-1}^{(k+1)}
$$

which is desired result.

(ii) From $|r|<1$, we have

$$
\begin{aligned}
\left\|\mathbb{F}_{r^{*}}^{(k)}\right\|_{E}^{2} & \geq \sum_{s=0}^{n-1}(n-s)\left|r^{n-s}\right|^{2}\left(\mathbb{F}_{s}^{(k)}\right)^{2}+\sum_{s=1}^{n-1} s\left|r^{n-s}\right|^{2}\left(\mathbb{F}_{s}^{(k)}\right)^{2} \\
& \geq n|r|^{2 n} \sum_{s=0}^{n-1}\left(\mathbb{F}_{s}^{(k)}\right)^{2} .
\end{aligned}
$$

From (5) and (4),

$$
\frac{|r|^{n}}{\sqrt{n}} \mathbb{F}_{n-1}^{(k+1)} \leq\left\|\mathbb{F}_{r^{*}}^{(k)}\right\|_{2}
$$

On the other hand, let the matrices $A^{(k)}$ and $B^{(k)}$ be defined by

$$
A^{(k)}=\left(\begin{array}{cccccc}
\mathbb{F}_{0}^{(k)} & 1 & 1 & \ldots & 1 & 1 \\
r & \mathbb{F}_{0}^{(k)} & 1 & \ldots & 1 & 1 \\
r^{2} & r & \mathbb{F}_{0}^{(k)} & \ldots & 1 & 1 \\
\vdots & \vdots & \vdots & & \vdots & \vdots \\
r^{n-1} & r^{n-2} & r^{n-3} & \ldots & r & \mathbb{F}_{0}^{(k)}
\end{array}\right)
$$

and

$$
B^{(k)}=\left(\begin{array}{cccccc}
\mathbb{F}_{0}^{(k)} & \mathbb{F}_{1}^{(k)} & \mathbb{F}_{2}^{(k)} & \ldots & \mathbb{F}_{n-2}^{(k)} & \mathbb{F}_{n-1}^{(k)} \\
\mathbb{F}_{n-1}^{(k)} & \mathbb{F}_{0}^{(k)} & \mathbb{F}_{1}^{(k)} & \ldots & \mathbb{F}_{n-3}^{(k)} & \mathbb{F}_{n-2}^{(k)} \\
\mathbb{F}_{n-2}^{(k)} & \mathbb{F}_{n-1}^{(k)} & \mathbb{F}_{0}^{(k)} & \ldots & \mathbb{F}_{n-4}^{(k)} & \mathbb{F}_{n-3}^{(k)} \\
\vdots & \vdots & \vdots & & \vdots & \vdots \\
\mathbb{F}_{1}^{(k)} & \mathbb{F}_{2}^{(k)} & \mathbb{F}_{3}^{(k)} & \ldots & \mathbb{F}_{n-1}^{(k)} & \mathbb{F}_{0}^{(k)}
\end{array}\right)
$$

That is, $\mathbb{F}_{r^{*}}^{(k)}=A^{(k)} \circ B^{(k)}$. Then we obtain

$$
r_{1}\left(A^{(k)}\right)=\max _{1 \leq i \leq n} \sqrt{\sum_{j=1}^{n}\left|a_{i j}^{(k)}\right|^{2}}=\sqrt{n-1}
$$


and

$$
c_{1}\left(B^{(k)}\right)=\max _{1 \leq j \leq n} \sqrt{\sum_{i=1}^{n}\left|b_{i j}^{(k)}\right|^{2}}=\sqrt{\sum_{s=0}^{n-1}\left(\mathbb{F}_{s}^{(k)}\right)^{2}} .
$$

From Lemma 1 and (4), we have

$$
\left\|\mathbb{F}_{r^{*}}^{(k)}\right\|_{2} \leq \sqrt{n-1} \mathbb{F}_{n-1}^{(k+1)}
$$

Thus we have

$$
\frac{|r|^{n}}{\sqrt{n}} \mathbb{F}_{n-1}^{(k+1)} \leq\left\|\mathbb{F}_{r^{*}}^{(k)}\right\|_{2} \leq \sqrt{n-1} \mathbb{F}_{n-1}^{(k+1)}
$$

\section{Conclusion}

In this paper we approximated lower and upper bounds of the spectral norms of geometric circulant matrices with the generalized Fibonacci and hyperharmonic Fibonacci numbers. If we take $p=1$ and $p=2$ in Theorem 1 , we obtain lower and upper bounds of the spectral norms of geometric circulant matrices with the Fibonacci and Pell numbers, respectively. Similarly if we take $p=1$ and $p=2$ in Theorem 2 , we obtain lower and upper bounds of the spectral norms of geometric circulant matrices with the Lucas and Pell-Lucas numbers, respectively.

In the future it may be possible that one can generalize our results to the Horadam, tribonacci and tribonacci-like sequences.

\section{Competing interests}

The authors declare that they have no competing interests.

\section{Authors' contributions}

Each of the authors contributed to each part of this work equally and read and approved the final version of the manuscript.

\section{Author details}

'Department of Mathematics, Bülent Ecevit University, Zonguldak, Turkey. ²Department of Mathematics, Gazi University, Ankara, Turkey.

\section{Acknowledgements}

The authors are grateful to two anonymous referees and the associate editor for their careful reading, helpful comments, and constructive suggestions, which improved the presentation of results.

Received: 11 August 2016 Accepted: 21 November 2016 Published online: 29 November 2016

\section{References}

1. Solak, S: On the norms of circulant matrices with the Fibonacci and Lucas numbers. Appl. Math. Comput. 160 125-132 (2005)

2. Kocer, EG, Mansour, T, Tuglu, N: Norms of circulant and semicirculant matrices with Horadam's numbers. Ars Comb. 85, 353-359 (2007)

3. Shen, SQ, Cen, JM: On the bounds for the norms of $r$-circulant matrices with Fibonacci and Lucas numbers. Appl. Math. Comput. 216, 2891-2897 (2010)

4. Bahsi, M: On the norms of $r$-circulant matrices with the hyperharmonic numbers. J. Math. Inequal. 10(2), 445-458 (2016)

5. Bahsi, M, Solak, S: On the norms of $r$-circulant matrices with the hyper-Fibonacci and Lucas numbers. J. Math. Inequal. 8(4), 693-705 (2014)

6. Jiang, Z, Zhou, Z: A note on spectral norms of even-order r-circulant matrices. Appl. Math. Comput. 250, 368-371 (2015)

7. Tuglu, N, Kizilateş, C: On the norms of circulant and $r$-circulant matrices with the hyperharmonic Fibonacci numbers. J. Inequal. Appl. 2015, 253 (2015)

8. Tuglu, N, Kizilates, C: On the norms of some special matrices with the harmonic Fibonacci numbers. Gazi Univ. J. Sci. 28(3), 447-501 (2015) 
9. Yazlik, Y, Taskara, N: On the norms of an $r$-circulant matrix with the generalized $k$-Horadam numbers. J. Inequal. Appl. 2013, 394 (2013)

10. He, C, Ma, J, Zhang, K, Wang, Z: The upper bound estimation on the spectral norm r-circulant matrices with the Fibonacci and Lucas numbers. J. Inequal. Appl. 2015, 72 (2015)

11. Tuglu, N, Kizilateş, C, Kesim, S: On the harmonic and hyperharmonic Fibonacci numbers. Adv. Differ. Equ. 2015, 297 (2015)

12. Bahsi, M: On the norms of circulant matrices with the generalized Fibonacci and Lucas numbers. TWMS J. Pure Appl. Math. 6(1), 84-92 (2015)

13. Horn, RA, Johnson, CR: Topics in Matrix Analysis. Cambridge University Press, Cambridge (1991)

Submit your manuscript to a SpringerOpen ${ }^{\odot}$ journal and benefit from:

- Convenient online submission

- Rigorous peer review

- Immediate publication on acceptance

- Open access: articles freely available online

- High visibility within the field

Retaining the copyright to your article

Submit your next manuscript at $\gg$ springeropen.com 